\title{
E-selectin Expression and Stimulation by Inflammatory Mediators are Developmentally Regulated during Embryogenesis
}

\author{
David S. Milstone, Peter E. O’Donnell, George Stavrakis, Richard M. Mortensen, \\ and Vannessa M. Davis
}

Vascular Research Division (DSM, PEO, GS, VMD), Department of Pathology, and Endocrine-Hypertension Division (RMM), Department of Medicine, Brigham and Women's Hospital, and Department of Pathology, Harvard Medical School, Boston, Massachusetts

\begin{abstract}
SUMMARY: Leukocyte recruitment during inflammation is specified, in part, by the spatial distribution and temporal regulation of endothelial adhesion molecules. In this study we investigated the developmental onset of E-selectin and intercellular adhesion molecule-1 (ICAM-1) basal expression and inducibility by inflammatory mediators as indices of lineage-restricted endothelial adhesion molecule expression. We studied both murine embryos and embryoid bodies (EB), derived from differentiated embryonic stem cells, to examine a broad range of endothelial ontogeny. Our results reveal that E-selectin and ICAM-1 are differentially regulated during development and that three stages define the ontogeny of the E-selectin-inducible response. The earliest endothelial lineage cells in Day 4 and Day 5 EB did not express E-selectin in the basal state or after stimulation. A second stage, observed between embryonic Day 9.5 (E9.5) and E11.5 to E12.5 in cultured embryo cells and transiently at Day 6 of EB differentiation, was characterized by basal expression that was not stimulated by inflammatory mediators. A third stage was characterized by both basal and inducible expression of E-selectin and was observed beginning at E12.5 to E13.5 in cultured embryo cells and at Day 7 in EB. In contrast ICAM-1 was stimulated at all of the embryonic stages examined and before the onset of E-selectin inducibility in both embryos and EB. E-selectin expression in embryos was also stimulated by introducing endotoxin into the embryonic, but not the maternal, peritoneum. This suggests that embryos are protected from inflammatory insults present in the maternal circulation. The developmentally regulated acquisition of E-selectin inducibility during embryogenesis likely involves changes in signal transduction cascades, transcription factors, and/or chromatin accessibility that specify inducible expression within the endothelial lineage and further restrict inducibility to particular endothelial subpopulations. (Lab Invest 2000, 80:943-954).
\end{abstract}

$R$ ecruitment of circulating leukocytes by activated vascular endothelium is important in acute and chronic inflammation and is specified, in part, by the expression of adhesion molecules including the selectins. Members of this family of cell surface, calciumdependent, glycoprotein lectins are expressed by endothelial cells (E-selectin), endothelium and megakaryocytes/platelets (P-selectin), and leukocytes (L-selectin) (reviewed in Vestweber and Blanks, 1999). A large body of evidence in both human and animal model systems shows that selectins regulate leukocyte recruitment and play essential roles in inflammation (Cotran and Mayadas-Norton, 1998; Luscinskas and Gimbrone, 1996). Patients with genetic abnormalities of those carbohydrate structures required for selectin counter-receptors suffer severe opportunistic

Received March 8, 2000.

These studies were supported by grants from The American Heart Association (92004370) and from the National Institutes of Health (HL54095 and HL30628).

Address reprint requests to: Dr. D. Milstone, Vascular Research Division, Department of Pathology, Brigham and Women's Hospital, LMRC 421, 221 Longwood Avenue, Boston, MA 02115. Fax: 617-975-0896; E-mail: milstone@rascal.med.harvard.edu infections caused by defective leukocyte recruitment (Etzioni et al, 1992; von Andrian et al, 1993). Mice that are genetically deficient in individual selectins also show specific abnormalities of leukocyte interactions with vascular endothelium (Arbones et al, 1994; Bullard et al, 1996; Kunkel and Ley, 1996; Labow et al, 1994; Ley et al, 1998; Mayadas et al, 1993; Milstone et al, 1998). Expression of the endothelial selectins at appropriate times and locations within the vasculature is thought to contribute to effective inflammatory responses, whereas altered expression patterns may contribute to the adverse consequences of various inflammatory pathologies.

E-selectin expression is precisely regulated in both time and space, suggesting that this adhesion molecule is uniquely important for leukocyte recruitment during the initial stages of inflammation. In support of this, we and others have shown, by intravital microscopy in genetically modified mice, that E-selectin is required in vivo for normal levels of leukocyte firm adhesion to acutely cytokine activated microvascular endothelium (Ley et al, 1998; Milstone et al, 1998) and for slow rolling of leukocytes on the vessel wall prior to adhesion (Kunkel and Ley, 1996). 
"Basal" expression of E-selectin is usually low and several inflammatory mediators, including tumor necrosis factor- $\alpha$ (TNF- $\alpha$ ), interleukin-1 (IL-1), CD40 ligand, and lipopolysaccharide (LPS) stimulate expression by activating transcription. Extensive analysis has established that this activation is mediated via an NF- $\kappa$ B-dependent promoter (Whelan et al, 1991; reviewed in Collins et al, 1995). Recently, Jun NH2terminal kinase (JNK)/stress-activated protein (SAP) kinases, presumably acting via phosphorylation of C-Jun and activation of the transcription factor C-Jun/ ATF-2, have been identified as additional critical mediators of E-selectin inducibility that may, at least in part, explain the differential responsiveness of different endothelial adhesion molecule genes in different pathologic, and potentially physiologic, settings (Min and Pober, 1997; Read et al, 1997). Interestingly, stimulation of E-selectin expression by TNF- $\alpha$, IL-1, or CD40 ligand renders endothelial cells transiently unresponsive to re-stimulation by the same, but not by a different, ligand. Such so-called homologous desensitization is paralleled by changes in JNK activation, suggesting that JNK may regulate this process (Karmann et al, 1996). Stimulated E-selectin expression is also usually transient because of inactivation of NF- $\kappa \mathrm{B}$ (Read et al, 1996), rapid degradation of E-selectin mRNA (Chu et al, 1994), and internalization of the mature protein from the cell surface (Kluger et al, 1997). This down-regulation is thought to contribute to the orderly resolution of inflammatory responses.

Stimulated E-selectin expression is not uniform throughout the vasculature but varies significantly among organs (Eppihimer et al, 1996; Hickey et al, 1999; DS Milstone et al, unpublished observations) and can be localized to physiologically important vascular structures, such as postcapillary venules of the human skin (Messadi et al, 1987; Petzelbauer et al, 1993). This spatial heterogeneity is also thought to play an important role in inflammation by restricting leukocyte recruitment to appropriate vascular locations (Hickey et al, 1999).

Although the mechanisms regulating activation of E-selectin expression have been studied intensively by many investigators, the factors that specify inducibility within the endothelial lineage, and that further restrict expression to particular endothelial subpopulations, have not been fully elucidated. NF- $\kappa$ B and JNK regulate transcription throughout the vasculature and elsewhere, suggesting that other molecular factors are required to impart lineage selectivity. Also, NF- $\kappa \mathrm{B}-$ responsive genes, such as ICAM-1 and vascular cell adhesion molecule-1 (VCAM-1), in addition to E-selectin, are expressed in distinct temporal and spatial patterns within the vasculature, suggesting additional layers of complexity. An inductive event, involving nonendothelial tissues and endothelium, and leading to cell lineage- and/or gene-specific expression, seems likely, given the characteristic and highly localized expression patterns observed.

As one approach to defining these lineage- and sublineage-restricted regulators, we have examined E-selectin expression and stimulation by inflammatory mediators during embryogenesis. The rationale for this approach is that understanding the initial specification of inducible expression during development might provide unique insights into the mechanisms regulating this process that would not be easily obtained solely from studies of mature vascular systems. Our results reveal that adhesion molecules in embryonic endothelium are inducible in response to inflammatory mediators, that E-selectin and ICAM-1 expression and stimulation are differentially regulated during embryogenesis, and that three distinct developmental stages are involved in maturation of the E-selectin-inducible response.

\section{Results}

\section{Endothelial Adhesion Molecule Genes Are Expressed during Embryogenesis}

To determine whether genes for endothelial-leukocyte adhesion molecules, important in inflammation, are expressed during embryogenesis, we performed ribonuclease protection assays (RPA) on total RNA isolated from murine embryos. The abundance of tie-2 mRNA, an endothelium-restricted transcript encoding a receptor for angiopoietins (Folkman and D'Amore, 1996; Sato et al, 1993), varied by less than $50 \%$ between E10.5 and E17.5, suggesting that representation of the endothelial lineage was relatively constant during this period (Fig. 1, A and B; see the "Materials and Methods" section for the methods of quantitation used throughout this manuscript). In contrast, the abundance of platelet-endothelial cell adhesion molecule (PECAM) mRNA, commonly taken as a marker of vascular endothelium, increased 3.6-fold between E9.5 and E17.5. However, megakaryocytes and platelets in adults also express PECAM, and cells of this lineage are present in embryonic murine liver after E12.5 (Matsumura and Sasaki, 1989). As a result, endothelial PECAM expression at E12.5 and later is probably less than the whole embryo expression levels shown in Figure 1. ICAM-1 mRNA, also expressed by some mature leukocytes, showed a similar pattern, increasing 3.3-fold between E10.5 and E17.5.

In contrast, E-selectin mRNA was detectable at E9.5 and was only $0.5 \%$ as abundant as ICAM- 1 mRNA at E10.5 (Fig. 1, A and B). Beginning by E12.5, expression increased a total of 17.5-fold at E17.5, becoming $2 \%$ to $3 \%$ as abundant as ICAM-1. P-selectin mRNA was also detected at E9.5 and increased 7.9-fold between E10.5 and E17.5, becoming approximately one-half as abundant as E-selectin mRNA at E15.5 and E17.5. These results reveal that mRNA for E-selectin, P-selectin, and ICAM-1 are expressed during embryogenesis in the absence of experimentally introduced inflammatory stimulation and that this "basal" expression of the endothelial selectins, and especially E-selectin, is significantly up-regulated relative to representation of the endothelial compartment between E12.5 and E17.5.

CD14 was also expressed in E9.5 embryos and increased 3- to 4-fold later in gestation (Fig. 1, A and 

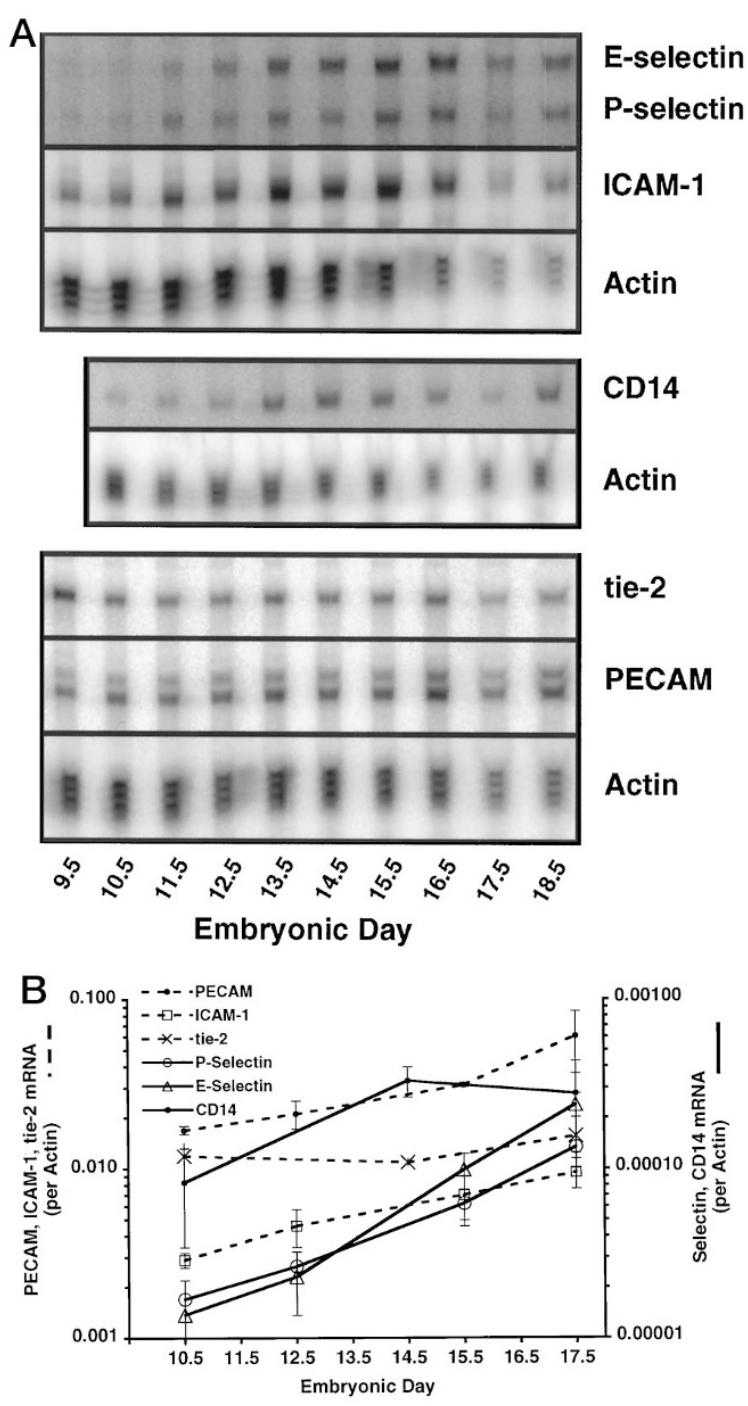

Figure 1.

Developmental time course of endothelial adhesion molecule, endothelial lineage marker, and CD14 expression during murine embryogenesis determined by RPA. A, Whole embryo RNA was analyzed from individual pups of timed litters using probes for the transcripts indicated. Protected fragments were detected by Phosphorlmager analysis. $B$, Expression of $\mathrm{E}$ - and P-selectin is differentially regulated during embryogenesis compared with other endothelium-restricted transcripts. The data are mean \pm SD derived from three or four independent embryos at each developmental time point analyzed as in A. E-selectin and P-selectin increased 17.9- and 7.9-fold, respectively, whereas tie-2, PECAM, ICAM-1, and CD14 increased only $\leq 1.5-, 3.6-$, 3.3-, and 3.4-fold, respectively, between E10.5 and E17.5. Note the dual logarithmic ordinates.

B). Either the cell surface or soluble form of this GPI-linked protein, along with circulating LPS-binding protein and an additional presumptive cell surface co-receptor, is required for efficient stimulation by endotoxin (reviewed in Fenton and Golenbock, 1998). Expression of CD14 suggests that, beginning at least at E9.5, the embryonic milieu may support inflammatory responses to bacterial endotoxin or other related products.

E-selectin expression is confined to vascular endothelium, whereas P-selectin is also expressed in megakaryocytes and platelets (Vestweber and Blanks,
1999). In contrast, although virtually all vascular endothelial cells in adult tissues express ICAM-1 (Carlos and Harlan, 1994; Cotran and Mayadas-Norton, 1998), this gene is also expressed by several nonendothelial cell types. Because we planned to compare selectin and ICAM-1 inducibility by inflammatory mediators, we identified ICAM-1-expressing cells during embryogenesis. Whole mount immunofluorescence staining, followed by confocal laser scanning microscopy (CLSM), revealed that ICAM-1 was largely coincident with PECAM protein in cranial vessels at E9.5 (Fig. 2) and in vertebral vessels at E9.5 and E10.5 (data not shown). Immunohistochemical analysis of whole mount preparations and cryostat sections also revealed that PECAM and ICAM-1 were restricted to the endothelium of most vascular structures between E9.5 and E11.5 (data not shown). Vessels staining for PECAM, but not ICAM-1, in Figure 2 probably reflect different expression levels of these proteins in vascular endothelial cells and/or greater detection sensitivity using anti-PECAM-FITC compared with anti-ICAM1-PE monoclonal antibodies. Immunohistochemistry did not reveal significant numbers of PECAM-positive/ ICAM-1-negative vessels in embryos. PECAM and ICAM-1 were also coexpressed by the extraembryonic yolk sac endothelium at E9.5 and later stages (DS Milstone, unpublished observations; Heyward et al, 1995). Taken together, these results demonstrate that ICAM-1 expression is restricted to vascular endothelium in embryos between E9.5 and E11.5.

\section{E-Selectin Is Inducible in Embryos by Inflammatory Mediators Introduced into the Embryonic, But Not the Maternal, Milieu}

Attempts to stimulate adhesion molecule expression in embryonic endothelium by ip injection of LPS into mothers were not successful. Expression of neither E-selectin nor P-selectin was stimulated in whole embryos at E10.5, E12.5, or E15.5, nor was it stimulated in embryos or kidney at E18.5 (Fig. 3, "maternal ip": $1.36 \pm 0.54, p=0.11$ for E-selectin and $1.16 \pm$ $0.31, p=0.16$ for P-selectin; mean $\pm \mathrm{SD}, n=5$ pairs of stimulated and unstimulated samples, with one pair each analyzed from E10.5, E12.5, and E15.5, and two analyzed from E18.5 embryos). In all cases the abundance of these transcripts was markedly stimulated (10- to 200-fold) in maternal tissues (not shown), confirming systemic distribution of the activation stimulus within the maternal compartment.

However, it was not clear whether this protocol successfully transmitted an activation stimulus to the embryos or, alternatively, the placental barrier functionally isolated the embryonic from the maternal compartments. Therefore, we measured the response to direct injection of LPS into the embryonic peritoneal cavity of E18.5 embryos that were maintained alive in utero in anesthetized mothers. This revealed significant stimulation of E-selectin and P-selectin mRNA (Fig. 3 "Embryo ip": $22.1 \pm 5.28, p=0.003$ and $4.09 \pm$ $0.79, p=0.003$, -fold stimulation, respectively; 
ICAM-1-PE

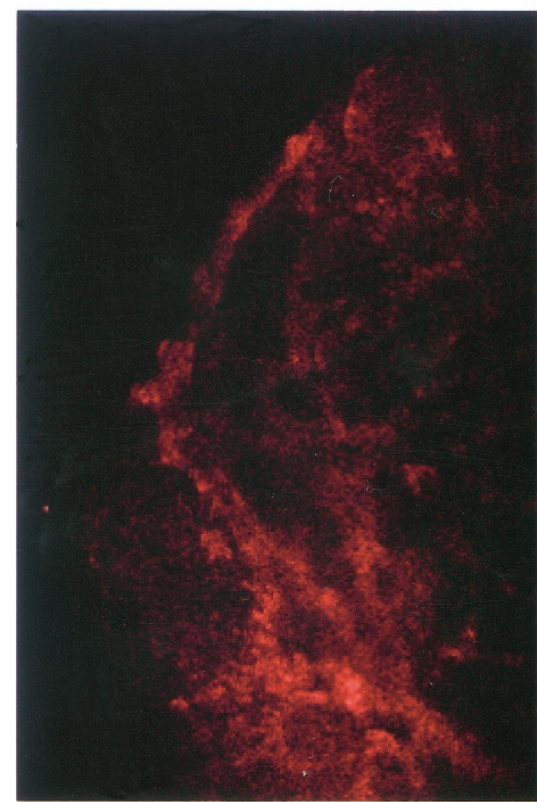

Merge

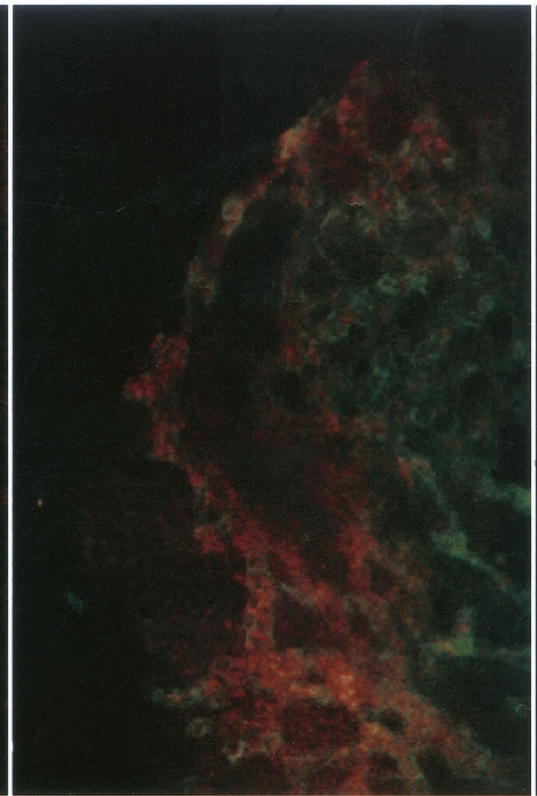

PECAM-FITC

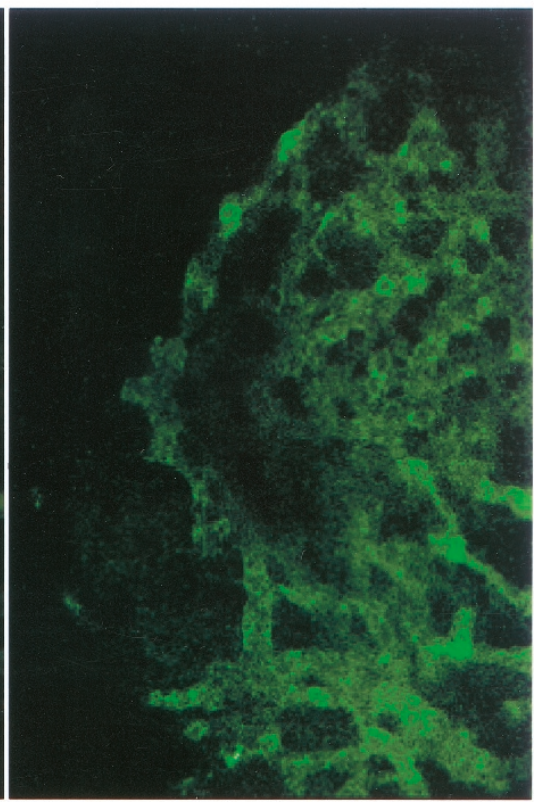

\section{Figure 2.}

ICAM-1 protein is co-localized with PECAM protein in vascular structures of E9.5 embryos. Embryos at E9.5 of development were stained by whole mount immunofluorescence simultaneously with anti-PECAM-FITC and anti-ICAM-1-PE. FITC and PE fluorescence were detected independently by CLSM. The cranium is shown. The merged image, shown in the center panel, confirms that ICAM-1 is expressed in vascular structures that also express PECAM. Intervertebral and other vessels of E9.5 and E10.5 embryos showed similar co-localization of ICAM-1 with PECAM protein (not shown).

mean $\pm \mathrm{SD}, n=4$ stimulated and 4 unstimulated samples) 2 hours after LPS administration. We also confirmed these in utero results by introducing LPS into the peritoneal cavity of E18.5 "embryos" immediately after Cesarean section delivery. This resulted in a $6.08 \pm 0.54$-fold increase $(p=0.02)$ of E-selectin mRNA (Fig. 3, "C-section ip"). In contrast, P-selectin expression was unchanged in these same samples (1.38 \pm 0.35 -fold stimulation ratio, $p=0.18$ ). Taken together, these results demonstrate that the endothelium of E18.5 embryos is capable of responding to inflammatory stimuli, but that the embryonic compartment is protected from such stimuli in the maternal circulation resulting from ip LPS injection in gravid females. The explanation for the apparent difference in P-selectin responsiveness at E18.5 between embryos stimulated in utero and those stimulated after Cesarean section delivery (Fig. 3) is not clear, but it may reflect the lower overall expression levels observed in the latter protocol.

\section{Inducibility of E-Selectin and ICAM-1 Are Differentially Regulated in Endothelium during Embryogenesis}

To determine when E-selectin responsiveness to cytokines and endotoxin is initially acquired during embryogenesis, we measured the expression of adhesion molecule mRNA in primary cultures of embryo cells stimulated with murine TNF- $\alpha$ (m-TNF $\alpha)$, murine mIL-1 $\alpha$ (mlL-1 $\alpha)$, murine IL-1 $\beta$ (mlL-1 $\beta)$, and LPS. These monolayer cultures ensured that stimuli could interact directly with the cells whose response was being tested, although indirect effects were also possible. The results demonstrated that both E-selectin and ICAM-1 mRNA were present, in the absence of exogenous stimulation, in cultures derived from all developmental stages between E9.5 and E13.5 (Fig. 4A), mirroring the expression observed in intact embryos (Fig. 1). However, TNF- $\alpha$ and LPS stimulated E-selectin expression only in cultures derived from E12.5 and older embryos $(2.75 \pm 0.74, p=0.002$, and $3.58 \pm 1.15, p=0.02$, -fold stimulation by mTNF- $\alpha$ and LPS, respectively), but not in cultures from earlier stages $(1.21 \pm 0.15, p=0.07$, and $1.09 \pm 0.07, p=$ 0.16 , -fold stimulation by mTNF- $\alpha$ and LPS, respectively; $p=0.002$ and $p=0.007$, respectively, compared with stimulation on and after E12.5; Fig. 4B and 4C). In contrast, TNF- $\alpha$ and LPS stimulated ICAM-1 expression equivalently at all embryonic stages tested $($ TNF- $\alpha: \leq \mathrm{E} 11.5=3.60 \pm 0.57, p=0.0001$, and $\geq$ $\mathrm{E} 12.5=3.94 \pm 1.67, p=0.002$, -fold stimulation; LPS: $\leq \mathrm{E} 11.5=4.94 \pm 1.67, p=0.01$, and $\geq \mathrm{E} 12.5=$ $4.60 \pm 1.55, p=0.003$, -fold stimulation). (Responses to $\mathrm{mlL}-1 \alpha, \mathrm{mlL}-1 \beta$, and LPS were not tested on cultured E9.5 cells because of the limited number of cells obtained.) Taken together, these results show that the initial inducibility of E-selectin by $\mathrm{mTNF}-\alpha$ and LPS does not occur until E12.5, even though the receptor systems, signal transduction mechanisms, and transcriptional response apparatuses for these mediators are functional and sufficient to stimulate expression of ICAM-1 at all stages examined. 


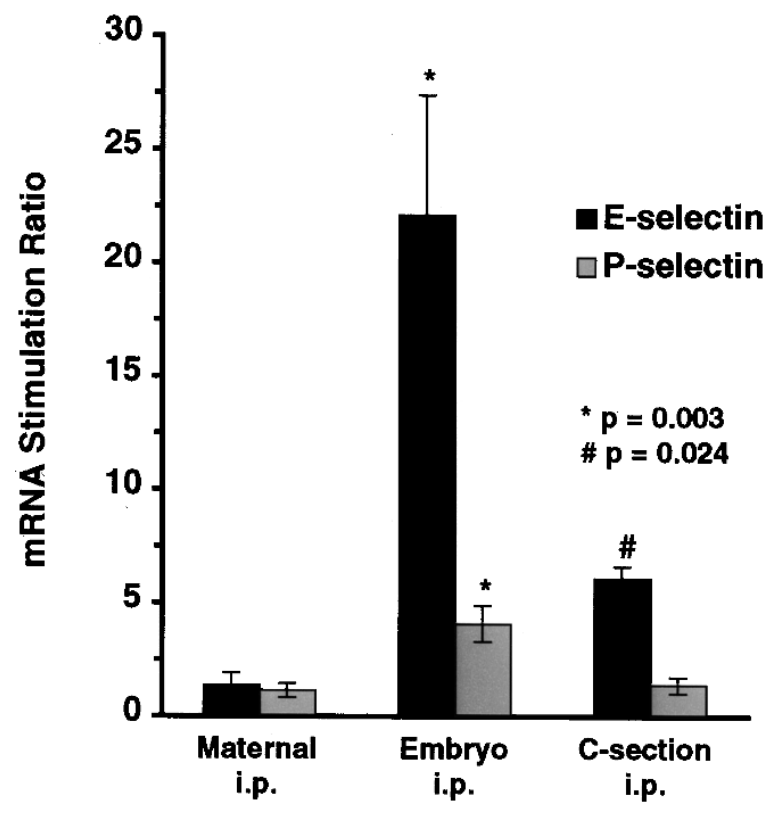

Figure 3.

Embryonic endothelium responds to inflammatory stimuli but is protected from stimuli present systemically in the mother. Maternal ip: LPS was injected into the peritoneal cavity of gravid females between E12.5 and E18.5. The data are mean \pm SD of 5 independent control and stimulated samples from E10.5, E12.5, E15.5, and E18.5. Embryo ip: LPS was injected into the peritoneal cavity of E18.5 embryos in utero as described in the "Materials and Methods" section. The data are mean \pm SD of 4 control and 4 stimulated embryos. C-section ip: LPS was injected into the peritoneal cavity of E18.5 embryos after Cesarean section delivery. The data are mean \pm sD from 3 control and 3 stimulated embryos. In each case embryos were harvested 2 hours after initial stimulation (see the "Materials and Methods" section) and analyzed by RPA. E-selectin is stimulated by introduction of LPS into the embryonic peritoneal cavity either in utero (Embryo ip) or after Cesarean section delivery (C-section ip) but not by introduction of LPS into the maternal peritoneal compartment (Maternal ip). P-selectin stimulation is statistically significant only after stimulation in utero (Embryo ip). ${ }^{*} p=0.003$ and $\# p=0.024$, stimulated vs unstimulated expression. Stimulated expression in the remainder of the experimental conditions was not significantly different from the corresponding control expression levels $(0.11 \leq p \leq 0.18)$.

\section{Embryoid Bodies Reveal an Additional Early Stage in the Ontogeny of E-Selectin Inducibility}

Analyzing cultured cells from embryos before E9.5 is problematic because of the difficulty of dissecting sufficient numbers of these small specimens free of maternal tissue. Therefore, to extend our analysis to earlier developmental stages, we examined EB derived by in vitro differentiation of murine embryonic stem cells (ES cells). When cultured in the absence of leukemia inhibitory factor and feeder cells, ES cells form EB that recapitulate the earliest stages of endothelial ontogeny. This includes the differentiation from mesoderm of angioblasts and committed endothelial lineage cells followed by the morphogenesis of vascular channels that are associated with hematopoietic colonies and are analogous to embryonic blood islands (Doetschman et al, 1993; Hirashima et al, 1999; Risau et al, 1988; Vittet et al, 1996; Wang et al, 1992; Yamaguchi et al, 1993). When exposed to inflammatory mediators, EB also express E-selectin mRNA with a stimulation sensitivity and time course similar to that observed in cultured endothelial cells (DS Milstone et al, unpublished observations). EB are thus an appropriate model system for analyzing endothelial responses to inflammatory mediators and offer significant advantages when examining the earliest stages of endothelial ontogeny.

Examination of EB at sequential developmental stages confirmed and extended the results observed in embryos. The vascular endothelial lineage was well represented, beginning at Day 4 , as reflected by vascular endothelial growth factor receptor 1 (VEGFR1 [flt-1]) and VEGFR2 (flk-1) expression, and underwent further differentiation as evidenced by tie-2 and PECAM expression beginning at Day 6 (Fig. 5A). In contrast, E-selectin mRNA was not detected at Day 4 or Day 5 of differentiation (Fig. 5B). A corresponding stage of endothelial ontogeny, lacking E-selectin expression, was not observed in embryos (Fig. 1) or cultured embryo cells (Fig. 4A), perhaps because these analyses did not include samples before E9.5. E-selectin was first expressed in EB, in the absence of exogenous inflammatory stimuli, at barely detectable levels at Day 6 (Fig. 5B), at least 2 full days after emergence of the vascular endothelial lineage. Inflammatory cytokines did not stimulate this earliest "basal" E-selectin expression $(0.93 \pm 0.64$-fold stimulation, $p=0.4$, Fig. $5 \mathrm{C}$ ) although low mRNA levels hampered quantitation. This was similar to the behavior of cultured cells from E9.5 to E11.5 embryos (Fig. 4, A to C). Stimulation by $\mathrm{mTNF}-\alpha$ and $\mathrm{mIL}-1 \beta$ was initially observed on Day 7 of EB differentiation (3.23 \pm 1.56 -fold stimulation, $p=0.01 ; p=0.03$ compared with stimulation at Day 6) and was increased in magnitude later (Fig. 5, B and C). This was similar to the expression pattern observed in cells cultured from E12.5 and E13.5 embryos (Fig. 4, A to C). In contrast, ICAM-1 mRNA was present, without stimulation, at all stages between Day 3 and Day 10 of EB differentiation and was stimulated by cytokines beginning at least by Day 6 (3.06 \pm 1.01 -fold stimulation, $p=0.01$ ) (Fig. 5 , B and C). ICAM-1 stimulation thus preceded stimulation of E-selectin in embryoid bodies, mirroring the results observed in cultured embryo cells.

\section{Discussion}

In these studies we characterized E-selectin and ICAM-1 expression and stimulation by inflammatory mediators during murine embryogenesis and in EB derived from ES cells, as an in vitro model of early endothelial differentiation. Our results reveal that the developmental onset of basal and inducible E-selectin and ICAM-1 expression are differentially regulated during embryogenesis, and we identify three specific stages in the maturation of the E-selectin inducible response. These results provide a foundation for further studies of the factors that dictate lineage- and sublineage-restriction of adhesion molecule inducibility in vascular endothelium.

The initial period (Stage 1, Fig. 6) of endothelial ontogeny was characterized by a lack of both basal and inducible E-selectin expression and was observed in Day 4 and Day 5 EB containing the earliest endo- 


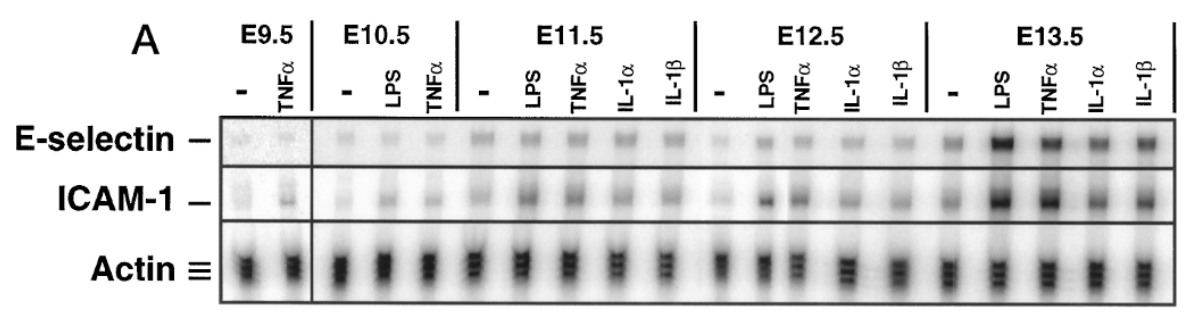

B
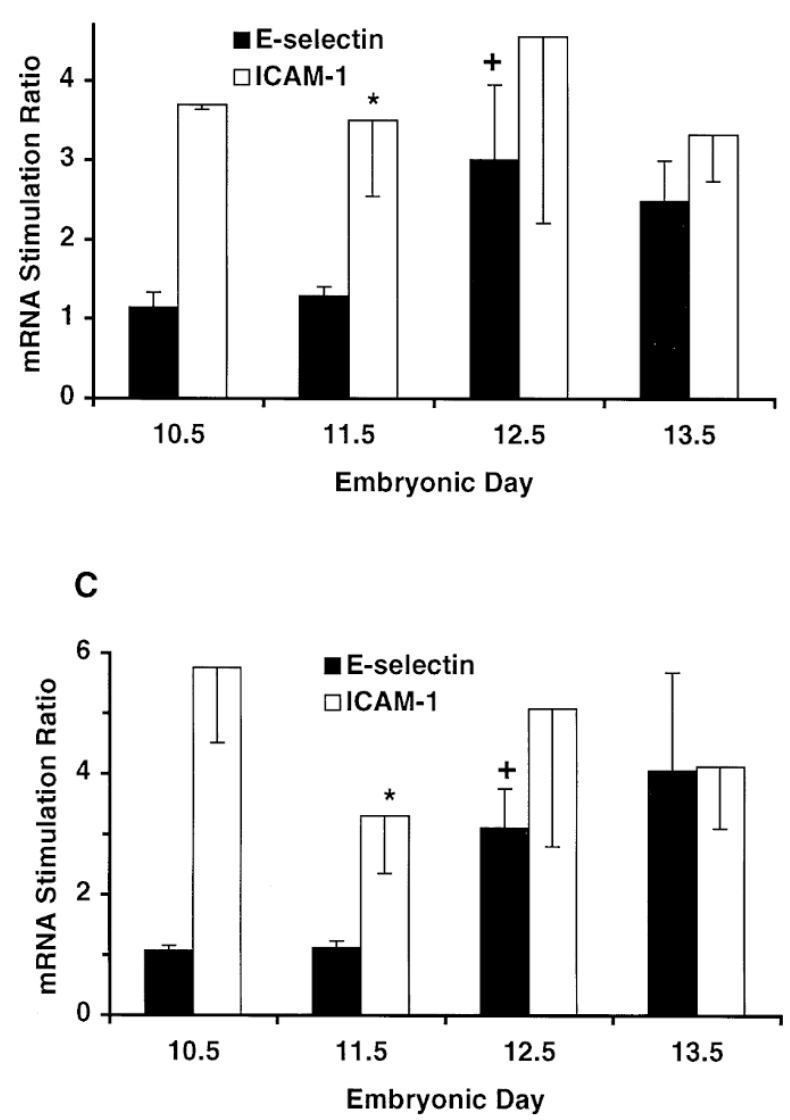

Figure 4.

Cytokine and LPS stimulation of E-selectin and ICAM-1 are differentially regulated during embryogenesis. $A$, Cells from embryos between E9.5 and E13.5 were cultured in vitro for 1 day and LPS, mTNF $\alpha$, mIL- $1 \alpha$, or mIL- $1 \beta$ were added for an additional 4 hours. E-selectin, ICAM-1, and actin mRNA were then assayed by RPA. $B$ and $C$, Quantitation (mean $\pm \mathrm{SD}$ ) of mTNF- $\alpha(B)$ and LPS $(C)$ stimulation of E-selectin and ICAM-1 mRNA from between 3 and 6 samples each from 3 independent experiments similar to that shown in $A$. mTNF- $\alpha$ stimulated E-selectin expression only in cells from E12.5 and E13.5 $(+p=0.03)$ but not from E10.5 or E11.5 $(p=$ 0.12 ) embryos, while ICAM-1 expression was stimulated at all stages examined ( $p=0.001$ and 0.015 , respectively). Correspondingly, ICAM-1 stimulation was significantly greater than E-selectin stimulation at $\mathrm{E} 11.5$ ( $\left.{ }^{*} p=0.004\right)$ but not at $\mathrm{E} 12.5$ or $\mathrm{E} 13.5(p=0.14)$. In addition, stimulation of adhesion molecule expression increased significantly between E11.5 and E12.5 for E-selectin (mTNF- $\alpha p=0.02$, LPS $p=0.02$ ) but not for ICAM-1 (mTNF- $\alpha p=0.20$, LPS $p=0.40$ ).

thelial lineage cells. This stage was not observed in whole embryos or cultured embryo cells, perhaps because we were not able to examine developmental stages before E9.5. Direct demonstration of Stage 1 in embryos may be difficult because of the low mRNA expression levels detected and the small number of endothelial lineage cells present before E9.5. During Stage 2, E-selectin was expressed in the basal state but was not stimulated by cytokines or LPS. This stage was most evident in cells cultured from embryos between E9.5 and E11.5, whereas a corresponding stage of differentiation was observed transiently in Day 6 EB. Stage 3 was defined by the onset of inducible E-selectin expression stimulated by inflammatory mediators and was equally prominent in embryos at E12.5 to E13.5, and in EB at Day 7 and later of differentiation. In contrast to this gradual maturation of the E-selectin inducible response, ICAM-1 was expressed in the basal state at all developmental stages examined and was inducible earlier during ontogeny than was E-selectin, both in cultured embryo cells and in EB. These results illustrate how the ES cell/EB system can be used to investigate stages of vascular development that would be difficult or impossible to assess using normal embryos. Cytokine stimulation of ICAM-1 and VCAM-1 has been re- 


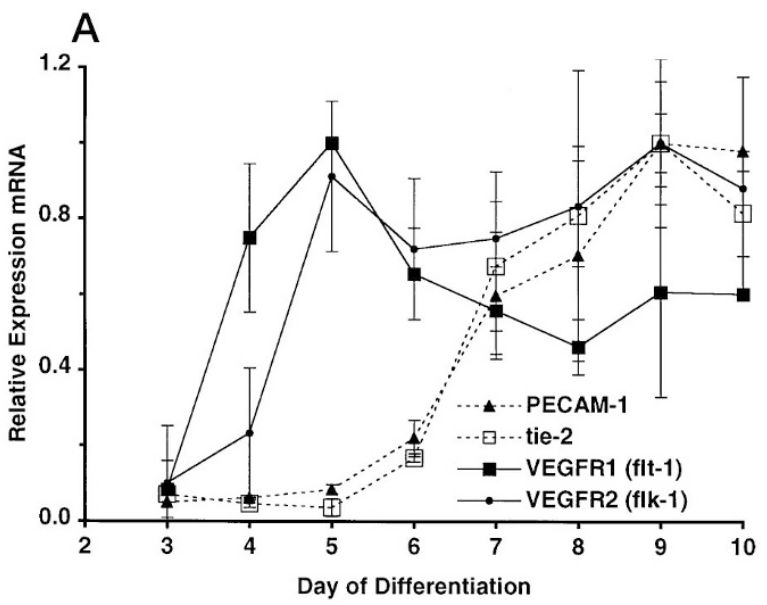

B
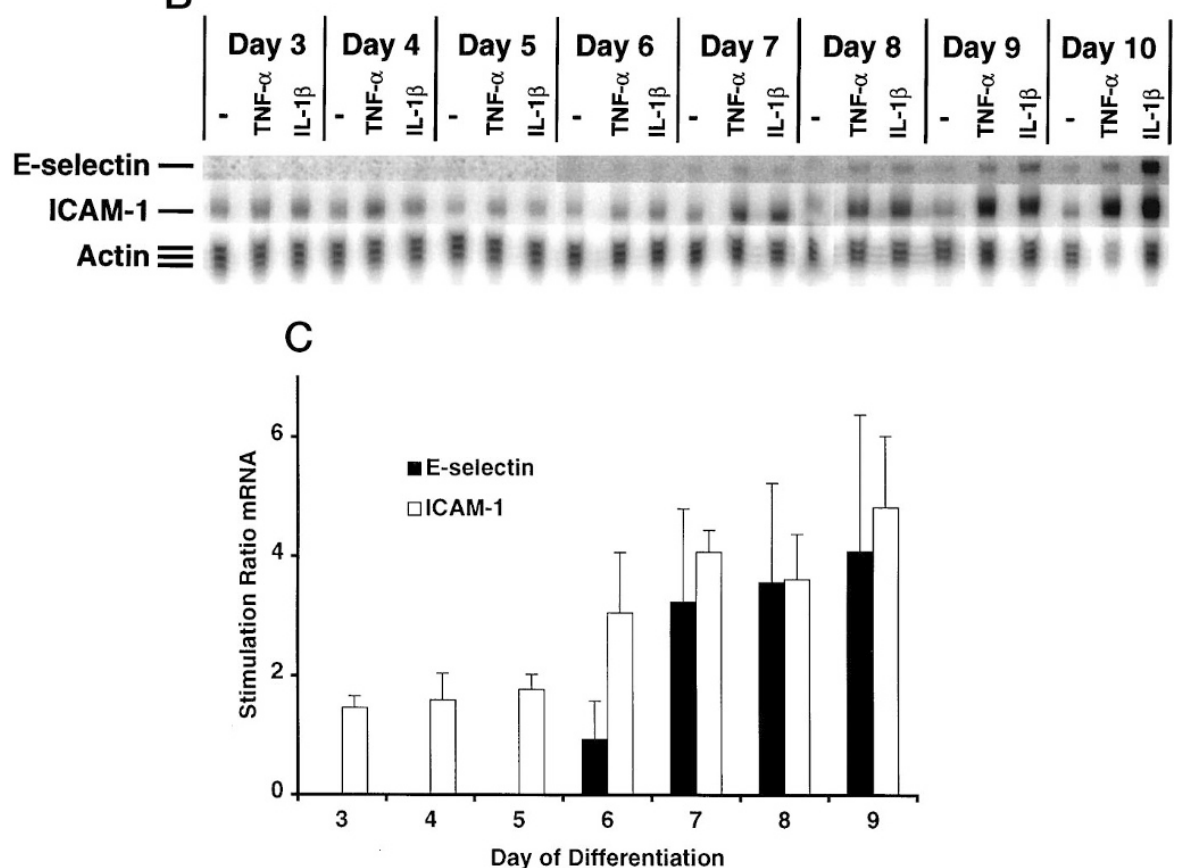

Figure 5.

Cytokine stimulation of E-selectin and ICAM-1 are differentially regulated during embryoid body differentiation. A, Embryoid bodies recapitulate early endothelial ontogeny. Endothelial lineage marker expression was analyzed by RPA and quantitated by Phosphorlmager analysis. The earliest stages of endothelial differentiation in EB are detected by VEGFR1 (flt-1) and VEGFR2 (flk-1) expression, beginning at Day 4, and by tie-2 and PECAM expression, beginning at Day 6. B, EB were differentiated for the times indicated and stimulated for 4 hours with mTNF- $\alpha$ or mIL-1 $\beta$. E-selectin, ICAM-1, and actin expression was then analyzed by RPA. $C$, Quantitation (mean $\pm \mathrm{SD}$ ) of mTNF- $\alpha$ stimulation of E-selectin and ICAM-1 mRNA from 3 replicates of the experiment shown in $B$. E-selectin was not detected prior to Day 6. Cytokines stimulated E-selectin expression only in Day 7 and later EB $(p \leq 0.01)$, but not in Day $6(p=0.4) \mathrm{EB}$. This is several days after emergence of the endothelial lineage on Day 4 and 1 day after early differentiation of endothelium on Day 6 (A). In contrast, ICAM-1 was stimulated strongly in Day 6 and later $\mathrm{EB}(p=0.01)$. Stimulation of adhesion molecule expression increased significantly between Day 6 and Day 7 of differentiation for $\mathrm{E}$-selectin $(p=0.03)$, but not for ICAM-1 $(p=0.17)$. The latter increased significantly between Day 5 and Day 6 of differentiation $(p=0.05)$. Correspondingly, ICAM-1 stimulation was significantly greater than E-selectin stimulation at Day $6(p=0.02)$, but not at Day 7 or later $(p \geq 0.2)$.

ported, and ICAM-1 has been localized to endothelial cells, in murine yolk sac and EB (Heyward et al, 1995). However, these authors did not investigate embryonic expression, the developmental acquisition of cytokine inducibility, or expression of E-selectin. Figure 6 summarizes the relationship between E-selectin and ICAM-1 basal expression and inducibility and other endothelial and vascular developmental landmarks. The differential regulation of E-selectin and ICAM-1 expression and inducibility revealed by our data may reflect either sequential developmental transitions within a single endothelial lineage or alternate differentiation pathways taken by cells of a common precursor population.

"Basal" expression of E-selectin in embryos in the absence of experimental stimulation (Fig. 1) may reflect either cell-intrinsic expression or activation by cell-extrinsic endogenous stimuli present in embryos. Genetic deficiency of TNF- $\alpha$ is accompanied by abnormal lymphoid organ development (Pasparakis et al, 


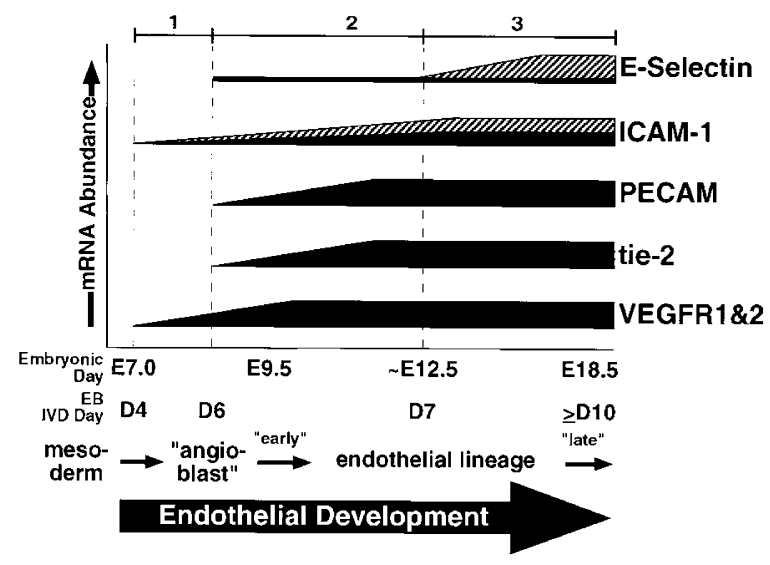

Figure 6.

E-selectin and ICAM-1 expression and stimulation by inflammatory mediators are differentially regulated during murine development and define later stages of endothelial ontogeny. The stages of endothelial lineage differentiation indicated at the top are represented in a time line that emphasizes transitions between functionally distinct developmental compartments and comparisons of normal embryos with EB derived by in vitro differentiation of ES cells. Data supporting the assignments before E9.5 in embryos are from the work of others. Data supporting the assignments in embryos at E9.5 and later and in EB are from the work presented in this report combined with the published work of others. Solid areas indicate basal expression. Hatched areas indicate expression stimulated by inflammatory mediators. IVD, in vitro differentiation.

1996) and rescues the embryonic lethal phenotype of NF- $\kappa$ B p65 deficiency (Doi et al, 1999), suggesting that this mediator is present and active during normal embryogenesis. TNF- $\alpha$ might, therefore, also be responsible for "basal" expression of endothelial adhesion molecules in embryos, although expression and function of this mediator has not been examined before E14.5 in mice (Doi et al, 1999). However, if "basal" adhesion molecule expression in vivo results from stimulation by TNF- $\alpha$, one might expect that this would lead to homologous desensitization (Karmann et al, 1996). Cultured cells from E9.5 to E11.5 embryos might then show augmented expression when stimulated with TNF- $\alpha$ after 24 hours of "quiescent" culture in vitro, during which the residual effects of in vivo exposure to TNF- $\alpha$ might diminish. This was not observed (Fig. 4) and is unlikely to reflect stimulusspecific homologous desensitization (Karmann et al, 1996) because these cells also did not respond to IL-1. However, it remains possible that TNF- $\alpha$ and/or other endogenous mediators contribute to the observed expression of E-selectin in the absence of experimental stimulation, especially in vivo. We have thus characterized this expression as "basal," but not necessarily "constitutive."

The initial acquisition of E-selectin inducibility appears distinct from several previously described developmental milestones of the endothelial lineage. Several endothelial-selective genes, such as VEGFR2 (flk-1), VEGFR1 (flt-1), tie-1, tie-2, and PECAM, are expressed as early as E7.0 to E8.5 of murine embryogenesis, when the vascular endothelial lineage is established and undergoes early differentiation (Folkman and D'Amore, 1996; Risau, 1997). In addition, spontaneous cardiac contractions, endothelium-lined blood vessels, and a functional circulatory system are prominent components of the developing embryo by E9.5 and reflect extensive vascular morphogenesis. In contrast, E-selectin was not stimulated until E12.5 to E13.5, several days after these differentiative and morphogenic events are well established. The precise temporal relationship between the acquisition of E-selectin inducibility and other embryologic events occurring near this stage in vivo is not clear, because our protocol included a 24-hour period of in vitro culture during which differentiation may have continued. For example, cultured E12.5 cells may more accurately represent the phenotype of E13.5 embryos.

Our experiments were designed to detect those endothelial populations that acquire E-selectin inducibility earliest in the development of the entire embryo. However, the transition to inducibility may occur at different developmental times, and to different degrees, in different embryonic vascular beds. We and others have observed that E-selectin inducibility varies quantitatively in different organs in adults (Eppihimer et al, 1996; Hickey et al, 1999), (DS Milstone et al, unpublished observations), and we are currently examining inducibility of E-selectin in specific embryonic organs. Determining when and how organ-specific differences arise during ontogeny may help identify the mechanisms regulating the spatial distribution of E-selectin inducibility, and functional heterogeneity in general, in mature endothelium.

It is possible that E-selectin was not inducible until E12.5 to E13.5 in embryos and until Day 7 in EB because the general mechanisms required for all responses to LPS, $\mathrm{mTNF} \alpha$, and $\mathrm{mlL}-1$ were not functional before these stages of development. The p75 and p55 TNF $\alpha$ receptors have, in fact, been shown to be absent in preimplantation murine embryos (Lachapelle et al, 1993). However, this explanation would require that the initial onset of functional responses to each of these mediators be coordinately and simultaneously regulated in endothelium. This seems unlikely. In addition, LPS, $\operatorname{mTNF} \alpha$, and $\mathrm{mlL}-1$ each stimulated ICAM-1, but not E-selectin expression in cultured E10.5 to E11.5 cells (Fig. 4, A to C). Because ICAM-1 expression is confined largely to vascular endothelium in embryos (Fig. 2), stimulated expression of ICAM-1 also probably occurs selectively in endothelial lineage cells. Therefore, during the developmental window between E9.5 and E11.5, E-selectin expression was not induced by several inflammatory stimuli that were sufficient to induce expression of ICAM-1.

The developmental onset of E-selectin inducibility thus appears to reflect the stage at which this genomic locus becomes responsive to a common expression mechanism, or to converging mechanisms, activated by several inflammatory mediators. This might be specified by expression of cell surface receptors in a previously nonresponsive subset of endothelial cells, by regulation of signal transduction or transcriptional regulatory mechanisms specifically required for E-selectin inducibility, or by enhanced accessibility of the E-selectin locus to a more promiscuous inducible 
expression mechanism. While induction in mature endothelium requires activation of $\mathrm{NF}-\kappa \mathrm{B}$, activating transcription factor-2 and high mobility group protein I (Y) (Whelan et al, 1991; reviewed in Collins et al, 1995), these are not sufficient on their own to confer either inducibility or endothelium-restricted expression. Other mechanisms must, therefore, be involved.

We hypothesize that such mechanisms operate only in endothelial lineage cells and that further restriction to specific vascular sites underlies the highly ordered anatomic localization of E-selectin inducibility observed in mature tissues. We expect that interactions with other localized cellular and/or noncellular elements of the developing vascular milieu are involved in specifying, and perhaps maintaining, the temporal and spatial regulation of E-selectin inducibility during ontogeny. Because JNK activity specifically regulates intricate patterns of E-selectin responsiveness to different inflammatory mediators (Karmann et al, 1996), it could also conceivably contribute to the initial onset of E-selectin inducibility during development.

We also observed that the developing endothelium is protected, presumably at the level of the placenta, from inflammatory stimuli present in the maternal milieu. LPS stimulates secondary release of numerous inflammatory mediators, including TNF- $\alpha$ from resident peritoneal macrophage, which are responsible for a significant portion of the resulting inflammatory sequelae (Amura et al, 1998). The placenta must thus present a barrier to several inflammatory agents, and this has significant practical implications for the health of mother and child. The existence of a placental barrier may also explain the observation that embryonic lethality in NF- $\kappa$ B p65-deficient embryos requires TNF- $\alpha$ in the fetal, but not the maternal, compartment (Doi et al, 1999). Alternatively, this could reflect low maternal expression of TNF- $\alpha$ although this cytokine is abundant in placenta in the absence of experimental inflammatory stimulation (Hunt, 1993). The fact that E-selectin is inducible in embryonic endothelium also implies that leukocyte recruitment may contribute to intrauterine inflammatory pathologies affecting the embryo. This would also require that circulating embryonic leukocytes be competent to undergo recruitment to sites of inflammation and endothelial activation. In this context, the lack of transplacental inducibility may be an important factor protecting embryos from systemic inflammatory insults suffered by gravid mothers.

In this report we show that E-selectin and ICAM-1 expression and stimulation are differentially regulated during embryogenesis and that three developmental stages are involved in maturation of the E-selectin inducible response. Understanding the cellular and molecular mechanisms that regulate the transitions between these stages, and how these transitions are differentially specified in different organs and vascular beds in embryos and in adults, may help explain how physiologically important endothelial functions are specified in healthy individuals and how they may be perturbed in disease.

\section{Materials and Methods}

\section{Cytokines and Endotoxin}

Murine TNF- $\alpha$ (mTNF- $\alpha)$, murine IL- $1 \alpha(\mathrm{mlL}-1 \alpha)$, and murine IL-1 $\beta$ (mlL-1 $\beta$ ) were purchased from Genzyme (Cambridge, Massachusetts) as purified recombinant proteins. Bacterial endotoxin (LPS from Escherichia coli Serotype 055:B5) was purchased from Sigma (Sigma L-2880, St. Louis, Missouri).

\section{Timed Murine Litters and Embryos and In Vivo Stimulation by IP Injection of Gravid Mothers}

All procedures involving animals conformed to institutional and federal guidelines and were approved by the appropriate institutional review boards. Matings were established between C57BL/6J mice (Jackson Laboratories, Bar Harbor, Maine) at 6 weeks to 6 months of age and maintained on a 7 am to 6 pm light cycle. Copulation plugs were detected between 9 am and $10 \mathrm{am}$. Noon of this day was designated E0.5. Expression of adhesion molecules was determined from litters obtained at noon on the appropriate days by euthanizing mothers using $\mathrm{CO}_{2}$ narcosis. Embryos were dissected free of all extraembryonic tissue in PBS, and RNA was isolated from whole embryo specimens and selected maternal organs. Ribonuclease protection assays (RPA) were performed as described below. Selected females at E10.5, E12.5, E15.5, and E18.5 were injected ip with $2 \mu \mathrm{g}$ LPS per gram body weight 2 hours before euthanasia.

\section{In Vivo Stimulation of E18.5 Embryos in Utero}

Gravid females at E18.5 were anesthetized with 2.5\% Avertin ( 0.15 to $0.17 \mathrm{ml} / \mathrm{g}$ body weight). Embryos were exposed surgically within the exposed maternal peritoneal cavity by opening the uterine wall and extraembryonic membranes, and $10 \mu \mathrm{l}$ PBS, with or without 2 $\mu \mathrm{g}$ LPS, was injected directly into the embryonic peritoneal cavity. Trypan blue $(0.1 \%, w / v)$ was included in these solutions to verify successful ip injection. These preparations were monitored continuously, warmed with a heat lamp, and moistened as needed with $0.9 \%$ saline solution. The uterine and umbilical circulations were maintained throughout the procedure. After 2 hours the embryos were dissected free of all extraembryonic tissue, RNA was isolated from whole embryo specimens and selected maternal organs, and RPA was performed as described below.

\section{In Vivo Stimulation of E18.5 Embryos after Cesarean Section Delivery}

Pups were delivered by Cesarean section essentially as described by Hogan et al (1994). Pregnant females were euthanized by $\mathrm{CO}_{2}$ narcosis and gravid uteri were immediately removed to warm PBS. Embryos were removed, the umbilical cords were severed, and the pups were warmed under a heat lamp. Respirations were stimulated by gentle pinching of the thorax and abdomen. Within 1 or 2 hours after delivery, all 
pups weighed between 1.1 and $1.3 \mathrm{~g}$, had established spontaneous respiratory activity sufficient for extrauterine survival, and were injected ip with $10 \mu \mathrm{l}$ PBS, with or without $2 \mu \mathrm{g}$ LPS. Pups were euthanized 2 hours later by $\mathrm{CO}_{2}$ narcosis, RNA was prepared from whole embryos and dissected kidneys, and RPA was performed as described below.

\section{Dissociation and In Vivo Culture of Embryo Cells}

All cell culture media components were purchased from Gibco/BRL (Rockville, Maryland). Fetal bovine serum was purchased from Hyclone Laboratories (Logan, Utah) and was screened for its ability to support in vitro differentiation of ES cells to vascular endothelial cells displaying a cytokine-responsive phenotype (see below). Embryos from timed gestations were dissected free of all maternal and extraembryonic fetal tissue and rinsed in Dulbecco's phosphate buffered saline (DPBS) with calcium and magnesium. The umbilical cords were severed at the embryonic abdominal wall, and the pooled embryos were minced with a sterile blade. The embryonic tissue fragments were transferred to $10 \mathrm{ml}$ of $0.05 \%$ trypsin and $0.53 \mathrm{~mm}$ ethylenediaminetetraacetic acid (EDTA) in Hank's Balanced Salt Solution, incubated at $37^{\circ} \mathrm{C}$ for 5 minutes with occasional swirling and trituration, and then without disturbance for 10 minutes. The upper $14 \mathrm{ml}$ of this digest was transferred to $10 \mathrm{ml}$ of complete medium (Dulbecco's Modified Eagle's Medium [DMEM] supplemented with $15 \%$ fetal bovine serum, $2 \mathrm{~mm}$ L-glutamine, and $0.1 \mathrm{~mm} 2$-mercaptoethanol). The remaining pellet was treated twice more with trypsin/ EDTA, and the released cells were pooled with the previous aliquots. Dissociated embryo cells were concentrated by centrifugation and resuspended in complete medium. The viability and extent of dissociation to single cells were quantitated by Trypan blue dye exclusion using a hemacytometer. Cells were plated at $4 \times$ $10^{5}$ viable cells $/ \mathrm{cm}^{2}$ on gelatin-coated, cell-culture grade plasticware. After 24 hours these cultures were washed several times with DPBS to remove nonadherent cells, fed with fresh complete medium, and stimulated with inflammatory mediators as described below.

\section{Embryonic Stem Cells and Embryoid Bodies}

CC1.2 ES cells (Robertson, 1987) were cultured without feeder cells in complete medium supplemented with recombinant murine leukemia inhibitory factor (LIF, ESGRO, $10^{3}$ units/ml, Gibco/BRL) to maintain the cells in an undifferentiated state (Hogan et al, 1994; Robertson, 1987). In vitro differentiation was initiated by culturing $10^{3}$ trypsin-dissociated ES cells in an inverted orientation on Fisher-brand bacterial petri dishes in $25 \mu$ l aliquots of complete medium lacking LIF. When the cells had coalesced into single aggregates (24 to 48 hours) approximately 100 of the resulting roughly spherical EB were cultured in suspension in $10 \mathrm{~cm}$ petri dishes in $10 \mathrm{ml}$ of complete medium, which was then changed every 1 to 2 days. Under these conditions $90 \%$ to $100 \%$ of EB develop
PECAM-expressing cells and islands of spontaneously contractile, electrically coupled cardiomyocytes (DS Milstone et al, unpublished observations).

\section{In Vitro Stimulation of Cultured Embryo Cells and EB}

LPS, $\operatorname{mTNF} \alpha, \mathrm{mIL}-1 \alpha$, or $\mathrm{mIL}-1 \beta$, each in $100 \mu \mathrm{l}$ of DPBS without $\mathrm{Mg}^{++}$or $\mathrm{Ca}^{++}$, or DPBS without $\mathrm{Mg}^{++}$ or $\mathrm{Ca}^{++}$alone, was added directly to embryo cell and EB cultures prepared as described above. The final concentrations of these mediators were $1 \mu \mathrm{g} / \mathrm{ml}, 20$ $\mathrm{ng} / \mathrm{ml}, 10 \mathrm{ng} / \mathrm{ml}$, and $10 \mathrm{ng} / \mathrm{ml}$, respectively. Cultures were harvested 4 hours later for RNA preparation. In preliminary experiments we determined that expression of E-selectin, P-selectin, and ICAM-1 mRNA was maximal after 2 hours of stimulation and remained relatively stable for at least an additional 2 hours. Thereafter, selectin mRNA slowly decreased in abundance, whereas ICAM-1 mRNA remained relatively stable for at least 24 hours.

\section{Isolation of RNA and Analysis by RPA}

Total RNA was isolated using guanidinium thiocyanate and cesium chloride gradients (Ausubel et al, 1994). Riboprobes labeled with $\alpha$-[ $\left.{ }^{32} \mathrm{P}\right]-$ rUTP were synthesized from partial cDNAs (see below) for the relevant transcripts cloned into the Bluescript phagemid (Stratagene, San Diego, California) or other appropriate cloning vector. Between 1 and $50 \mu \mathrm{g}$ of total cellular RNA per sample were analyzed by RPA using a commercially available kit (Ambion, Austin, Texas). Specific activities of the probes were varied by including unlabeled rUTP during the in vitro transcription reaction to facilitate analysis of multiple transcripts in a single hybridization reaction. Protected fragments were detected and quantitated using a Molecular Dynamics Phosphorlmager, model 425E, and ImageQuant software. Baseline-corrected and integrated peak areas were further corrected for nucleotide composition of the protected fragments and specific activity of the radiolabeled ribonucleotide substrates before performing additional calculations and statistical analyses. All values were normalized to the actin expression level in the same sample determined simultaneously. These corrected and normalized expression levels were then compared using Student's $t$ test. A $p$ value of $\leq 0.05$ designated statistical significance.

\section{Hybridization Probes}

The riboprobes used for murine E-selectin and P-selectin have been described (Milstone et al, 1998). DNA templates used for additional riboprobes were prepared from cloned cDNA for PECAM, CD14, and VEGFR2 (flk-1) (cDNA generously supplied by Drs. William Muller, Richard Ulevitch, and John Flanagan, respectively) and by RT-PCR from murine tissue RNA for ICAM-1, tie-2, and VEGFR1 (flt-1). The pfu polymerase (Stratagene, San Diego, California) was used for polymerase chain reaction (PCR) to minimize amplification errors, and the fidelity of all cloned products was verified by DNA sequence analysis (Sequenase 
Table 1. Hybridization Probes

\begin{tabular}{lrrrl}
\hline \multicolumn{1}{c}{ Transcript } & Start nt & End nt & $\begin{array}{r}\text { Protected Fragment } \\
(\mathrm{nt})\end{array}$ & \multicolumn{1}{c}{ Citation of Sequence } \\
\hline PECAM & 2250 & 2475 & 226 & PNAS (1992)90:5569-5573. \\
ICAM-1 & 13 & 239 & 227 & EMBO J (1989)8:2889-2896. \\
VEGFR1 (flt-1) & 20 & 182 & 193 & Oncogene (1993)8:2293-2298. \\
VEGFR2 (flk-1) & 2761 & 3033 & 273 & Cell (1993)72:835-846. \\
tie-2 & 2025 & 2301 & 277 & PNAS (1993)90:9335-9358. \\
CD14 & 120 & 449 & 233 & J Immunol (1990)145:331-336. (97 nt IVS) \\
\hline
\end{tabular}

2.0; United States Biochemical Corporation, Cleveland, Ohio). The probes were as shown in Table 1.

\section{Immunohistochemistry and Immunofluorescence}

Purified rat monoclonal antibodies specific for murine ICAM-1 and PECAM, directly coupled, or not, to fluorescein isothiocyanate (FITC) or phycoerythrin (PE), were purchased from Pharmingen (San Diego, California). Immunohistochemistry on cryostat sections was performed as described (Milstone et al, 1998). For whole mount immunofluorescence analysis, samples were fixed in MeOH:DMSO 4:1 (v:v) for 0.5 to 4 hours at $4{ }^{\circ} \mathrm{C}$ and processed as described (Hogan et al, 1994). Primary conjugated antibodies were applied at $4{ }^{\circ} \mathrm{C}$ for 12 to 16 hours each in the presence of detergents to assist penetration. Extensive washing and protein blocking steps were used before the addition of reagents.

\section{Confocal Microscopy}

Confocal laser scanning microscopy (CLSM) was performed with a Zeiss fluorescence microscope (Zeiss, Welwyn Garden City, United Kingdom) and BioRad MRC-6000 SLC (Richmond, California) apparatus and image analysis system. Additional analysis of confocal image files was accomplished using NIH Image, Adobe Photoshop, CLSM Artist, and Oncor Corporation software on Macintosh PowerPC 7100/66 and 8500/120 computers and a Compac $75 \mathrm{MHz}$ Pentium computer. Image file stacks were "flattened" to create two-dimensional representations of the cumulative signal present throughout the imaged volumes.

\section{Acknowledgements}

We thank Dr. David Conner for assistance with ES cell culture techniques and Dr. Michael Gimbrone for critical review of the manuscript before submission for publication.

\section{References}

Amura CR, Silverstein R, and Morrison DC (1998). Mechanisms involved in the pathogenesis of sepsis are not necessarily reflected by in vitro cell activation studies. Infect Immun 66:5372-5378.

Arbones ML, Ord DC, Ley K, Ratech H, Maynard-Curry C, Otten G, Capon DJ, and Tedder TF (1994). Lymphocyte homing and leukocyte rolling and migration are impaired in L-selectin-deficient mice. Immunity 1:247-260.
Ausubel FM, Brent R, Kingston RE, Moore DD, Seidman JG, Smith JA, and Struhl K (1994). Current protocols in molecular biology. New York: Current Protocols.

Bullard DC, Kunkel EJ, Kubo H, Hicks MJ, Lorenzo I, Doyle NA, Doerschuk CM, Ley K, and Beaudet AL (1996). Infectious susceptibility and severe deficiency of leukocyte rolling and recruitment in $\mathrm{E}$-selectin and $\mathrm{P}$-selectin double mutant mice. J Exp Med 183:2329-2336.

Carlos TM and Harlan JM (1994). Leukocyte-endothelial adhesion molecules. Blood 84:2068-2101.

Chu W, Presky DH, Swerlick RA, and Burns DK (1994). Alternatively processed human E-selectin transcripts linked to chronic expression of E-selectin in vivo. J Immunol 153: 4179-4189.

Collins T, Read MA, Neish AS, Whitley MZ, Thanos D, and Maniatis T (1995). Transcriptional regulation of endothelial cell adhesion molecules: NF-kappa-B and cytokine-inducible enhancers [Review]. FASEB J 9:899-909.

Cotran RS and Mayadas-Norton T (1998). Endothelial adhesion molecules in health and disease. Pathol Biol (Paris) 46:164-170.

Doetschman T, Shull M, Kier A, and Coffin JD (1993). Embryonic stem cell model systems for vascular morphogenesis and cardiac disorders. Hypertension 22:618-629.

Doi TS, Marino MW, Takahashi T, Yoshida T, Sakakura T, Old LJ, and Obata $Y$ (1999). Absence of tumor necrosis factor rescues RelA-deficient mice from embryonic lethality. Proc Natl Acad Sci USA 96:2994-2999.

Eppihimer MJ, Wolitzky B, Anderson DC, Labow MA, and Granger DN (1996). Heterogeneity of expression of E- and P-selectins in vivo. Circ Res 79:560-569.

Etzioni A, Frydman M, Pollack S, Avidor I, Phillips ML, Paulson JC, and Gershoni-Baruch R (1992). Brief report: Recurrent severe infections caused by a novel leukocyte adhesion deficiency. N Engl J Med 327:1789-1792.

Fenton MJ and Golenbock DT (1998). LPS-binding proteins and receptors. J Leukoc Biol 64:25-32.

Folkman J and D'Amore PA (1996). Blood vessel formation: What is its molecular basis [Comment]? Cell 87:1153-1155.

Heyward SA, Dubois-Stringfellow N, Rapoport R, and Bautch VL (1995). Expression and inducibility of vascular adhesion receptors in development. FASEB J 9:956-962.

Hickey MJ, Kanwar S, McCafferty DM, Granger DN, Eppihimer MJ, and Kubes P (1999). Varying roles of E-selectin and $\mathrm{P}$-selectin in different microvascular beds in response to antigen. J Immunol 162:1137-1143. 
Hirashima M, Kataoka H, Nishikawa S, and Matsuyoshi N (1999). Maturation of embryonic stem cells into endothelial cells in an in vitro model of vasculogenesis. Blood 93:12531263.

Hogan B, Beddington R, Costantini F, and Lacy E (1994). Manipulating the mouse embryo: A laboratory manual, 2nd edition. Plainview, NY: Cold Spring Harbor Laboratory Press.

Hunt JS (1993). Expression and regulation of the tumour necrosis factor-alpha gene in the female reproductive tract. Reprod Fertil Dev 5:141-153.

Karmann K, Min W, Fanslow WC, and Pober JS (1996). Activation and homologous desensitization of human endothelial cells by CD40 ligand, tumor necrosis factor, and interleukin 1. J Exp Med 184:173-182.

Kluger MS, Johnson DR, and Pober JS (1997). Mechanism of sustained E-selectin expression in cultured human dermal microvascular endothelial cells. J Immunol 158:887-896.

Kunkel EJ and Ley K (1996). Distinct phenotype of E-selectindeficient mice. E-selectin is required for slow leukocyte rolling in vivo. Circ Res 79:1196-1204.

Labow MA, Norton CR, Rumberger JM, Lombard-Gillooly KM, Shuster DJ, Hubbard J, Bertko R, Knaack PA, Terry RW, Harbison ML, and et al (1994). Characterization of E-selectindeficient mice: Demonstration of overlapping function of the endothelial selectins. Immunity 1:709-720.

Lachapelle MH, Miron P, Hemmings R, Falcone T, Granger L, Bourque J, and Langlais J (1993). Embryonic resistance to tumour necrosis factor-alpha mediated cytotoxicity: Novel mechanism underlying maternal immunological tolerance to the fetal allograft. Hum Reprod 8:1032-1038.

Ley K, Allietta M, Bullard DC, and Morgan S (1998). Importance of E-selectin for firm leukocyte adhesion in vivo. Circ Res 83:287-294.

Luscinskas FW and Gimbrone MA Jr (1996). Endothelialdependent mechanisms in chronic inflammatory leukocyte recruitment. Annu Rev Med 47:413-421.

Matsumura G and Sasaki K (1989). Megakaryocytes in the yolk sac, liver and bone marrow of the mouse: A cytometrical analysis by semithin light microscopy. J Anat 167:181-187.

Mayadas TN, Johnson RC, Rayburn H, Hynes RO, and Wagner DD (1993). Leukocyte rolling and extravasation are severely compromised in P-selectin-deficient mice. Cell 74: 541-554.

Messadi DV, Pober JS, Fiers W, Gimbrone MA Jr, and Murphy GF (1987). Induction of an activation antigen on postcapillary venular endothelium in human skin organ culture. J Immunol 139:1557-1562.

Milstone DS, Fukumura D, Padgett RC, O'Donnell PE, Davis VM, Benavidez OJ, Monsky WL, Melder RJ, Jain RK, and Gimbrone MA Jr (1998). Mice lacking E-selectin show normal numbers of rolling leukocytes but reduced leukocyte stable arrest on cytokine-activated microvascular endothelium. Microcirculation 5:153-171.

Min W and Pober JS (1997). TNF initiates E-selectin transcription in human endothelial cells through parallel TRAFNF-kappa B and TRAF-RAC/CDC42-JNK-C-Jun/ATF2 pathways. J Immunol 159:3508-3518.
Pasparakis M, Alexopoulou L, Episkopou V, and Kollias G (1996). Immune and inflammatory responses in TNF alphadeficient mice: A critical requirement for TNF alpha in the formation of primary B cell follicles, follicular dendritic cell networks and germinal centers, and in the maturation of the humoral immune response [Comments]. J Exp Med 184: 1397-1411.

Petzelbauer P, Bender JR, Wilson J, and Pober JS (1993). Heterogeneity of dermal microvascular endothelial cell antigen expression and cytokine responsiveness in situ and in cell culture. J Immunol 151:5062-5072.

Read MA, Neish AS, Gerritsen ME, and Collins T (1996). Postinduction transcriptional repression of E-selectin and vascular cell adhesion molecule-1. J Immunol 157:3472-3479.

Read MA, Whitley MZ, Gupta S, Pierce JW, Best J, Davis RJ, and Collins T (1997). Tumor necrosis factor alpha-induced E-selectin expression is activated by the nuclear factorkappaB and c-JUN N-terminal kinase/p38 mitogen-activated protein kinase pathways. J Biol Chem 272:2753-2761.

Risau W (1997). Mechanisms of angiogenesis. Nature 386: 671-674.

Risau W, Sariola H, Zerwes HG, Sasse J, Ekblom P, Kemler $R$, and Doetschman $T$ (1988). Vasculogenesis and angiogenesis in embryonic-stem-cell-derived embryoid bodies. Development 102:471-478.

Robertson EJ (1987). Teratocarcinomas and embryonic stem cells: A practical approach. Oxford: IRL Press Ltd, 254.

Sato TN, Qin Y, Kozak CA, and Audus KL (1993). Tie-1 and tie-2 define another class of putative receptor tyrosine kinase genes expressed in early embryonic vascular system [published erratum appears in Proc Natl Acad Sci USA 90:12056]. Proc Natl Acad Sci USA 90:9355-9358.

Vestweber D and Blanks JE (1999). Mechanisms that regulate the function of the selectins and their ligands. Physiol Rev 79:181-213.

Vittet D, Prandini MH, Berthier R, Schweitzer A, MartinSisteron H, Uzan G, and Dejana E (1996). Embryonic stem cells differentiate in vitro to endothelial cells through successive maturation steps. Blood 88:3424-3431.

von Andrian UH, Berger EM, Ramezani L, Chambers JD, Ochs HD, Harlan JM, Paulson JC, Etzioni A, and Arfors KE (1993). In vivo behavior of neutrophils from two patients with distinct inherited leukocyte adhesion deficiency syndromes. J Clin Invest 91:2893-2897.

Wang R, Clark R, and Bautch VL (1992). Embryonic stem cell-derived cystic embryoid bodies form vascular channels: An in vitro model of blood vessel development. Development 114:303-316.

Whelan J, Ghersa P, Hooft van Huijsduijnen R, Gray J, Chandra G, Talabot F, and DeLamarter JF (1991). An NF kappa B-like factor is essential but not sufficient for cytokine induction of endothelial leukocyte adhesion molecule 1 (ELAM-1) gene transcription. Nucleic Acids Res 19:2645-2653.

Yamaguchi TP, Dumont DJ, Conlon RA, Breitman ML, and Rossant J (1993). flk-1, an flt-related receptor tyrosine kinase is an early marker for endothelial cell precursors. Development 118:489-498. 